\title{
A New Member of Cardiovirus: Unknown Pathogenicity to Humans
}

\section{Yoshiro Ohara* and Toshiki Himeda}

Department of Microbiology, Kanazawa Medical University School of Medicine Ishikawa, Japan

Members of the family Picornaviridae are nonenveloped viruses with a single-stranded RNA genome of positive polarity. 'Fields Virology' reads that the family contains many important human and animal pathogens and is composed of nine genera; aphthovirus, cardiovirus, enterovirus, erbovirus, hepatovirus, kobugvirus, parechovirus, rhinovirus, and teschovirus in the fifth edition [1]. The genus Cardiovirus contains Encephalomyocarditis virus (EMCV) and Theiler's murine encephalomyelitis virus (TMEV) and has long been believed to infect mainly rodents. However, in 2007, Saffold virus (SAFV) is identified from the stool sample with fever of unknown origin as a novel human cardiovirus [2]. Subsequently, SAFV was isolated from nasal and stool specimens from infants presenting with respiratory or gastrointestinal symptoms. In addition, the virus was isolated from the cerebrospinal fluid specimen of patient with aseptic meningitis. Since some seroepidemiological studies demonstrated that the seroprevalence to SAFV reaches $>90 \%$ in older than 24 months children and adults in several countries, SAFV is thought to be spread world-wide. Although several epidemiological studies have been reported up to now, they have failed to provide a clear answer of the relationship between SAFV and human diseases [3].

Animal experiments have been carried out in order to study the pathogenicity of SAFV, although SAFV does not infect rodents naturally. The experiments of different two groups suggest that SAFV is neurotropic in mice. Of special note is the presence of inflammation in the spinal cord white matter, because the closely related mouse cardiovirus, TMEV, causes a demyelinating central nervous system disease that resembles multiple sclerosis [3]. Therefore, the recombination between SAFV and TMEV, which could alter the host range, will be a serious problem to humans. However, Himeda et al. reported in the other issue of J Plant Pathol Microbiol that the recombination of capsid proteins between SAFV and TMEV did not occur [4]. Another important issue is that the major viral load following SAFV intraperitoneal inoculation is the pancreas. Several viruses in the family Picornaviridae, particularly Coxsackie B viruses have long been implicated in the etiology of type I diabetes. In addition, EMCV is known to induce pancreatitis and type I diabetes in rodents [3]. Recently, several European groups started the study to investigate the relationship between SAFV and type I diabetes. The answer to this issue awaits the data from them.

From these observations, the pathogenicity of SAFV still remains unclear, although the potential pathogenicity of SAFV to humans is thought to be varied (respiratory, gastrointestinal and neurological diseases and type I diabetes etc.). In order to clarify the pathogenicity of SAFV, the further epidemiological studies including the data of healthy persons as a control group is required. In addition, the researches of viral factors involved in the pathogenicity of SAFV using a reverse genetic technique [5] are needed. Furthermore, the identification of the receptor(s) for SAFV infection is also important in order to establish the transgenic mice as a novel animal model to study the pathogenicity of SAFV [3].

\section{References}

1. Vincent R. Racaniello VR (2007) Picornaviridae: the viruses and their replication, 795-838. In Knipe DM, Howley PM (ed.), Fields Virology, 5th Ed. Lippincott Williams \& Wilkins, a Wolters Kluwer Business, Philadelphia.

2. Jones MS, Lukashov VV, Ganac RD, Schnurr DP (2007) Discovery of a nove human picornavirus in a stool sample from a pediatric patient presenting with fever of unknown origin. J Clin Microbiol 45: 2144-2150.

3. Himeda T, Ohara $Y$ (2011) Saffold virus, a novel human cardiovirus with unknown pathogenicity. $J$ Virol published online ahead of print on 23 Nov. 2011

4. Himeda T, Nojiri M, Okuwa T, Muraki Y, Ohara Y (2011) Reverse genetic analysis of the recombination in Theilovirus based on the infectious cDNA clones. J Plant Pathol Microbiol in press

5. Himeda T, Hosomi T, Asif N, Shimizu H, Okuwa T, et al. (2011) The preparation of an infectious full-length cDNA clone of Saffold virus. Virol J 8: 110.
*Corresponding author: Yoshiro Ohara, M.D, Department of Microbiology, Kanazawa Medical University School of Medicine, 1-1 Uchinada, Ishikawa 9200293, Japan, Tel: 81-76-286-221; Fax: 81-76-286-3961; E-mail: ohara@kanazawamed.ac.jp

Received December 07, 2011; Accepted December 08, 2011; Published December 10, 2011

Citation: Ohara Y, Himeda T (2011) A New Member of Cardiovirus: Unknown Pathogenicity to Humans. J Plant Pathol Microbiol 2:e101. doi:10.4172/2157$7471.1000 \mathrm{e} 101$

Copyright: ( 2011 Ohara Y, et al. This is an open-access article distributed under the terms of the Creative Commons Attribution License, which permits unrestricted use, distribution, and reproduction in any medium, provided the original author and source are credited. 